\title{
Article
}

\section{Graphene oxide synthesis by facile method and its characterization}

\author{
Farman ullah Khan ${ }^{1}$, Sajid Mahmood ${ }^{2}$, Zaheer Ahmad ${ }^{1, *}$, Tariq Mahmood ${ }^{3}$ and Zamir Ahmad Nizami ${ }^{4}$ \\ 1 Department of Chemistry, University of Wah, Wah Cantt 47040, Pakistan. \\ 2 Department of Chemistry, Division of Science and Technology, University of Education, Lahore 54000, Pakistan. \\ 3 Nano Sciences and Technology Department, National Centre for Physics, QAU, Islamabad 45320, Pakistan. \\ 4 Department of Chemistry, University of Sargodha,Sub Campus Bhakkar, Pakistan. \\ * Correspondence: dr.zaheer.ahmad@uow.edu.pk; Tell: +92-3322569362
}

Received: 10 March 2019; Accepted: 21 May 2019; Published: 30 June 2019.

\begin{abstract}
The graphene and graphene oxide are latest and advanced materials with wide applications in environment, medical applications, industries, defense applications. We have synthesized graphene oxide from graphite flakes by modifying Hummer method in which we used $\mathrm{NaNO}_{2}$ instead of $\mathrm{NaNO}_{3}$. Then we characterized our samples with X-Ray Diffractometry (XRD), Scanning-Electron Microscopy (SEM) and Fourier-Transform Infrared-Spectroscopy (FT-IR). These results confirmed the formation of graphene oxide also through this process. This graphene oxide can further be used for future applications in wastewater treatment and biomedical applications.
\end{abstract}

Keywords: Graphene oxide, nanochemistry.

\section{Introduction}

$\mathbf{T}$ he importance of graphene and its composites is increasing due to their enlarged applications in the solar cells, catalysis and hydrogen storage. They are used in sensors, nanoelectronics and nanocomposites [1-5]. Graphene is the single layer of graphite. All the carbon atoms of graphene are $\mathrm{sp}^{2}$ hybridized. They are arranged in a honey comb lattice like structure. Graphene is carbon allotrope. It has hexagonal atomic structure and therefore hexagonal nanoparticles are easily adjusted in their layers. Their hydrogels have crosslinked nanoshheet structure [6]. The nanocomposite of $\mathrm{TiO}_{2}$ and graphene is exhibiting large surface area, higher activity and the $\mathrm{N}_{2}$ selection and at low temperature high resistance towards water and sulfur dioxide and also, high redox activity by which the catalytic reaction is favored [7]. Several studies have shown that metal-metal oxide-graphene have triple junction structure formation. They act like the electro-catalyst for the oxygen reduction for the applications in the PEM fuel cell. The tests performed showed that the durability of Pt became better than Pt on graphene sheets but also from Pt electro-catalysts which are supported on Carbon material, for example; Carbon nano-tubes [8].

Aijun et al. have worked on graphene and $g-C_{3} N_{4}$ interface by the state of art of the hybrid functional DFT procedure and incorporated the long range dispersion correction. They for first time revealed that electronic coupling is strong at graphene and $g-C_{3} N_{4}$ interface [9]. Xuefeng et al. reviewed the most recent research on the graphene materials and their antimicrobial activities. They also discussed the physiochemical properties of graphene materials, their experimental surrounding and selected mocro-organisms and the interaction between them to further explore the controversial antimicrobial properties [10]. Wang et al. prepared metal oxide and graphene nanocomposite. They studied the properties of various metal oxide and graphene nanocomposite for the super capacitors and also for electrochemical catalysis [11]. Vats et al. synthesized nanocomposite of pristine-graphene with the palladium. They used swollen liquids crystal as the soft template. They observed that the catalytic activity of the nanocomposite is better than the Pd-RGO nanocomposite in one of the hydrogenation reaction of nitro-phenol and microwave assisted carbon-carbon coupling reaction which was sixteen times higher [12]. Kian et al. studied the graphene and the molecules like graphene for their role in the solar cells [13]. Mousavi et al. prepared PV-RGO and used as anodic battery material. That composite showed high areal, volumetric and the current density [14]. 
Meng et al. synthesized nanocomposite of silver nanoparticles decorated with graphene by chemical reduction process with assistance by supercritical $\mathrm{CO}_{2}\left(\mathrm{ScCO}_{2}\right)$. They studied tribological properties of the nanocomposite. It showed the high lubricating function of the graphene and the roughness of surface of the sliding ball is reduced and prevents direct interaction [15]. Tengfei et al. worked on the synthesis of nanocomposite of iron oxide and silver nanoparticles with graphene. They compared the Ag nanoparticles with this nanocomposite. They found that the nanocomposite was showing enhanced antibacterial activity towards the Gram negative bacteria, E. coli and the Gram positive bacteria which is S. aureus [16]. Li et al. synthesized graphene sheet, polymeric carbon-nitride nanocomposite which work as metal free catalyst for activation of oxygen for the selective oxidation of the sec. carbon-hydrogen bonds of saturated hydrocarbons like cyclohexane. It was observed to be the most stable catalyst and having high chemoselectivity for the sec. $\mathrm{C}-\mathrm{H}$ bond of different saturated alkane [17]. Liwen et al. synthesized a nanocomposite of Graphene Oxide and Sulfur for the immobilization of sulfur in cathodic material of Li-S cells [18]. Deepak et al. synthesized Graphene Oxide and studied its antibacterial activities [19]. Zheng et al. prepared reduced graphene oxide by reducing graphene oxide by help of a reducing agent (caffeic acid) which was having high carbon-oxygen ratio i.e (??) which is best reduced graphene oxide prepared from green reducing ragent [20]. Hongmei et al. used solvothermal method and developed magnetic-reduced graphene oxide (MRGO) nanocomposite. The nanocomposite was having high removal efficiency. It was observed that the M-RGO composite is effective adsorbent used for the removal of dyes pollutant [21]. Myungwoo et al. reviewed the graphene oxide synthesis which was developed through chemical vapor deposition method and studied its application [22]. Zhang et al. developed reduced graphene oxide and $\mathrm{NiO}$ nanocomposite which was used for the absobtion of the chromium ion $(\mathrm{Cr}(\mathrm{VI}))$. The composite showed maximum adsoption capacity of chromium ion at $\mathrm{PH}=4$ and $\mathrm{T}=25^{0} \mathrm{C}$ which was higher than any other reported so far [23]. Marcano et al. prepared graphene oxide by modification in the Hummer method as they excluded $\mathrm{NaNO}_{3}$. They increased $\mathrm{KMnO}_{4}$ and used $\mathrm{H}_{3} \mathrm{PO}_{4}$ and $\mathrm{H}_{2} \mathrm{SO}_{4}$ mixture in the ratio of 1:9 which showed improvement in the oxidation process of graphene [24].

\section{Method and materials}

We took $2 \mathrm{~g}$ of graphite and $\mathrm{NaNO}_{2}$ and mixed in about $100 \mathrm{~mL}$ of $\mathrm{H}_{2} \mathrm{SO}_{4}$ (conc.) in $1000 \mathrm{~mL}$ volumetric flask and put in ice-bath. The mixture was continuously stirred on hot plate for about 1 hour and then added $6 \mathrm{~g}$ of $\mathrm{KMNO}_{4}$ slowly. Then removed ice bath and kept the mixture under stirring for 2 days. About $90 \mathrm{~mL}$ of distilled water was added to this and brown color appeared. The solution was stirred continuously. Then $15 \mathrm{~mL}$ of hydrogen peroxide was added to this solution and yellow color appeared. We washed this mixture with $10 \% \mathrm{HCl}$. Then centrifuged at 5000rpm for 5 minute. This filtrate was decanted. The remaining (graphene oxide) was dried at $110^{\circ} \mathrm{C}$ and then calcined for 3 hours at $550^{\circ} \mathrm{C}$ in muffle furnce. The graphene oxide thus obtained was grind and characterized for further analysis.

\section{Results and discussions}

For the characterization of prepared nanoparticles XRD is one of the best technique. It characterizes the purity andphase of the nanomaterial. XRD gives detail of diffraction angle, the interlayer spacing and mainly the crystallite size. The XRD used in our work was Bruker D8 advance. Figure 1 shows XRD pattern of graphite used in this work. Figure 2 is showing wide-angle XRD peak of our prepared graphene oxide. In graphene oxide XRD spectra, the major peak at $11.6^{\circ}$ and two weak peaks at $2 \theta=43^{0}$ and $2 \theta=45^{0}$ clearly describe the graphite structure [22,25]. We characterized the graphene oxide by the Field-emission scanning-electron microscopy (FESEM) for their surface morphology. Figure 3 shows FESEM image of graphene oxide at different magnifications. It is clear from the FESEM image of GO that their layers are smooth in appearance [25]. The prepared graphene oxide was characterized by FT-IR for functional-groups checking. Figure 4 show the FT-IR spectra of the graphene oxide. The major peak at about $3500 \mathrm{~cm}^{-1} \mathrm{~s}$ hows the presence of hydroxyl $(\mathrm{OH})$ groups [25]. Some peaks were also observed in fingerprint region at $750 \mathrm{~cm}^{-1}-600 \mathrm{~cm}^{-1}$ which may be the bending vibrations due to $\mathrm{C}-\mathrm{O}$, and $\mathrm{C}-\mathrm{O}-\mathrm{C}$ bending vibrations.

\section{Conclusion}

In summary, we synthesized Graphene Oxide from graphite powder by using $\mathrm{NaNO}_{2}$ instead of $\mathrm{NaNO}_{3}$. The resultant product obtained was characterized through different analytical techniques like, XRD, FESEM, 


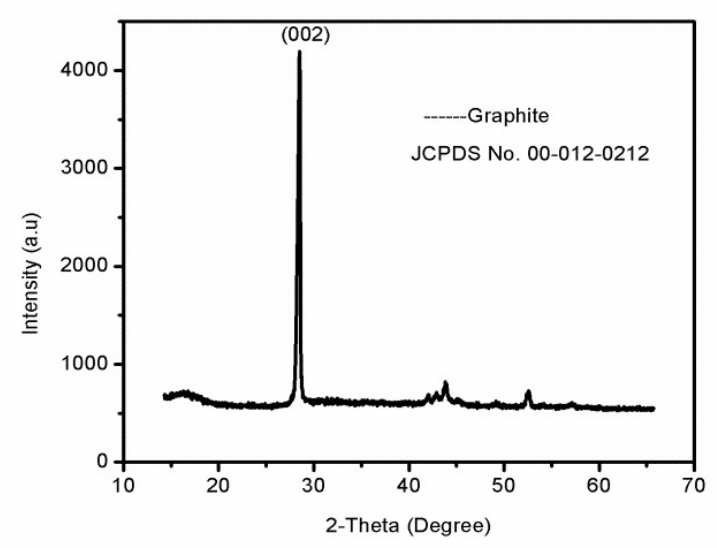

Figure 1. XRD pattern of graphite

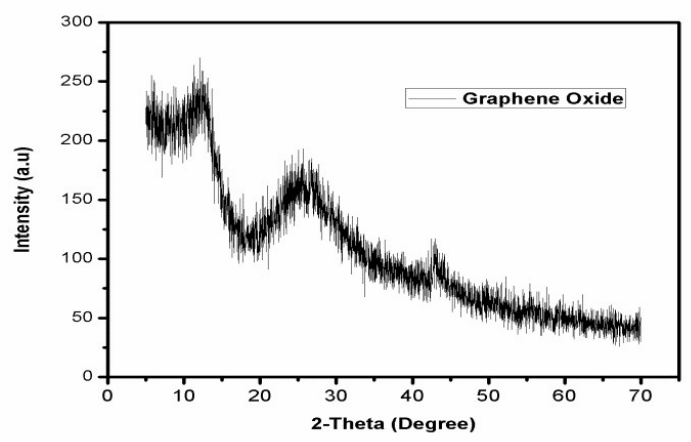

Figure 2. XRD pattern of graphene oxide

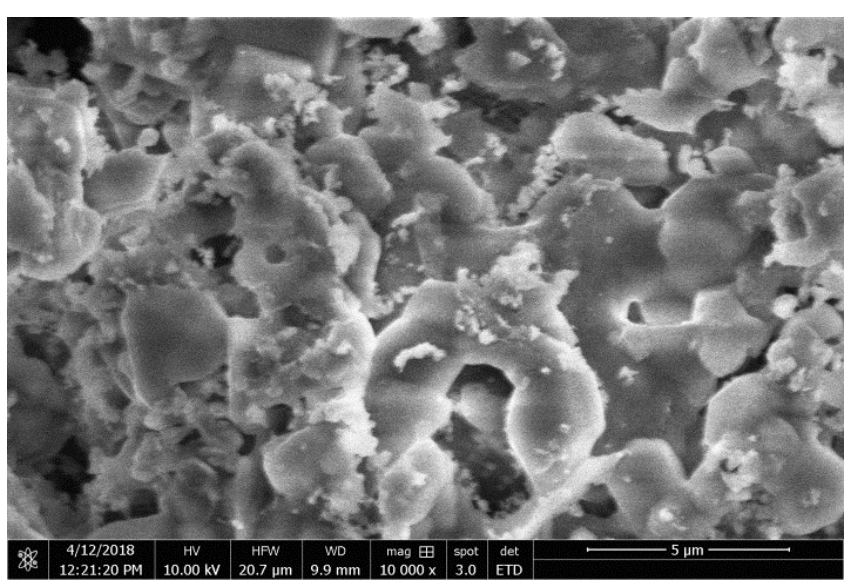

Figure 3. SEM image of Graphene Oxide

FT-IR. The obtained result was matched with literature and this was confirmed that the graphene oxide has formed. The oxidation of graphene can be increased by using $\mathrm{NaNO}_{2}$. This can be used for different purposes specially as adsorbant of heavy metals.

Author Contributions: All authors contributed equally to the writing of this paper. All authors read and approved the final manuscript.

Conflicts of Interest: "The authors declare no conflict of interest." 


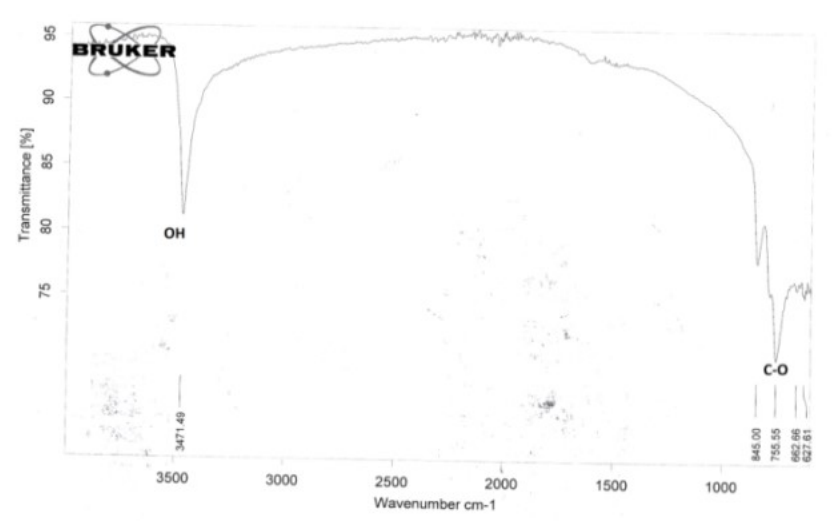

Figure 4. FT-IR spectra of Graphene oxide

\section{References}

[1] Chen, H., \& Carroll, K. C. (2016). Metal-free catalysis of persulfate activation and organic-pollutant degradation by nitrogen-doped graphene and aminated graphene. Environmental pollution, 215, 96-102.

[2] Cho, K. Y., Seo, H. Y., Yeom, Y. S., Kumar, P., Lee, A. S., Baek, K. Y., \& Yoon, H. G. (2016). Stable 2D-structured supports incorporating ionic block copolymer-wrapped carbon nanotubes with graphene oxide toward compact decoration of metal nanoparticles and high-performance nano-catalysis. Carbon, 105, 340-352.

[3] Kwon, S. N., Jung, C. H., \& Na, S. I. (2016). Electron-beam-induced reduced graphene oxide as an alternative hole-transporting interfacial layer for high-performance and reliable polymer solar cells. Organic Electronics, 34, 67-74.

[4] Xia, G., Tan, Y., Wu, F., Fang, F., Sun, D., Guo, Z., \& Yu, X. (2016). Graphene-wrapped reversible reaction for advanced hydrogen storage. Nano Energy, 26, 488-495.

[5] Park, C. S., Yoon, H., \& Kwon, O. S. (2016). Graphene-based nanoelectronic biosensors. Journal of Industrial and Engineering Chemistry, 38, 13-22.

[6] Jiao, T., Guo, H., Zhang, Q., Peng, Q., Tang, Y., Yan, X., \& Li, B. (2015). Reduced graphene oxide-based silver nanoparticle-containing composite hydrogel as highly efficient dye catalysts for wastewater treatment. Scientific reports, $5,11873$.

[7] Lu, X., Song, C., Chang, C. C., Teng, Y., Tong, Z., \& Tang, X. (2014). Manganese Oxides Supported on TiO2-Graphene Nanocomposite Catalysts for Selective Catalytic Reduction of NO X with NH3 at Low Temperature. Industrial $\mathcal{E}$ Engineering Chemistry Research, 53(29), 11601-11610.

[8] Kou, R., Shao, Y., Mei, D., Nie, Z., Wang, D., Wang, C., ... \& Wang, Y. (2011). Stabilization of electrocatalytic metal nanoparticles at metal-metal oxide-graphene triple junction points. Journal of the American Chemical Society, 133(8), 2541-2547.

[9] Du, A., Sanvito, S., Li, Z., Wang, D., Jiao, Y., Liao, T., ... \& Smith, S. C. (2012). Hybrid graphene and graphitic carbon nitride nanocomposite: gap opening, electron-hole puddle, interfacial charge transfer, and enhanced visible light response. Journal of the American Chemical Society, 134(9), 4393-4397.

[10] Zou, X., Zhang, L., Wang, Z., \& Luo, Y. (2016). Mechanisms of the antimicrobial activities of graphene materials. Journal of the American Chemical Society, 138(7), 2064-2077.

[11] Wang, D., Kou, R., Choi, D., Yang, Z., Nie, Z., Li, J., .. \& Liu, J. (2010). Ternary self-assembly of ordered metal oxide-graphene nanocomposites for electrochemical energy storage. ACS nano, 4(3), 1587-1595.

[12] Vats, T., Dutt, S., Kumar, R., \& Siril, P. F. (2016). Facile synthesis of pristine graphene-palladium nanocomposites with extraordinary catalytic activities using swollen liquid crystals. Scientific reports, 6, 33053.

[13] Loh, K. P., Tong, S. W., \& Wu, J. (2016). Graphene and graphene-like molecules: prospects in solar cells. Journal of the American Chemical Society, 138(4), 1095-1102.

[14] Beladi-Mousavi, S. M., Sadaf, S., Mahmood, A. M., \& Walder, L. (2017). High Performance Poly (viologen)-Graphene Nanocomposite Battery Materials with Puff Paste Architecture. ACS nano, 11(9), 8730-8740.

[15] Meng, Y., Su, F., \& Chen, Y. (2016). Supercritical fluid synthesis and tribological applications of silver nanoparticle-decorated graphene in engine oil nanofluid. Scientific reports, $6,31246$.

[16] Tian, T., Shi, X., Cheng, L., Luo, Y., Dong, Z., Gong, H., ... \& Liu, Z. (2014). Graphene-based nanocomposite as an effective, multifunctional, and recyclable antibacterial agent. ACS applied materials $\mathcal{E}$ interfaces, 6(11), 8542-8548. 
[17] Li, X. H., Chen, J. S., Wang, X., Sun, J., \& Antonietti, M. (2011). Metal-free activation of dioxygen by graphene/g-C3N4 nanocomposites: functional dyads for selective oxidation of saturated hydrocarbons. Journal of the American Chemical Society, 133(21), 8074-8077.

[18] Ji, L., Rao, M., Zheng, H., Zhang, L., Li, Y., Duan, W., ... \& Zhang, Y. (2011). Graphene oxide as a sulfur immobilizer in high performance lithium/sulfur cells. Journal of the American Chemical Society, 133(46), 18522-18525.

[19] Gupta, D. K., Rajaura, R. S., \& Sharma, K. (2015). Synthesis and Characterization of Graphene Oxide Nanoparticles and their Antibacterial Activity. Suresh Gyan Vihar University International Journal of Environment, Science and Technology, 1(1), 16-24.

[20] Bo, Z., Shuai, X., Mao, S., Yang, H., Qian, J., Chen, J., ... \& Cen, K. (2014). Green preparation of reduced graphene oxide for sensing and energy storage applications. Scientific reports, $4,4684$.

[21] Sun, H., Cao, L., \& Lu, L. (2011). Magnetite/reduced graphene oxide nanocomposites: one step solvothermal synthesis and use as a novel platform for removal of dye pollutants. Nano Research, 4(6), 550-562.

[22] Son, M., \& Ham, M. H. (2017). Low-temperature synthesis of graphene by chemical vapor deposition and its applications. FlatChem, 5, 40-49.

[23] Zhang, K., Li, H., Xu, X., \& Yu, H. (2018). Synthesis of reduced graphene oxide/NiO nanocomposites for the removal of $\mathrm{Cr}$ (VI) from aqueous water by adsorption. Microporous and Mesoporous Materials, 255, 7-14.

[24] Marcano, D. C., Kosynkin, D. V., Berlin, J. M., Sinitskii, A., Sun, Z., Slesarev, A., ... \& Tour, J. M. (2010). Improved synthesis of graphene oxide. ACS nano, 4(8), 4806-4814.

[25] Atchudan, R., Edison, T. N. J. I., Perumal, S., Karthikeyan, D., \& Lee, Y. R. (2016). Facile synthesis of zinc oxide nanoparticles decorated graphene oxide composite via simple solvothermal route and their photocatalytic activity on methylene blue degradation. Journal of Photochemistry and Photobiology B: Biology, 162, 500-510. 